\title{
Discontinuous Galerkin Time-Domain Modeling of Graphene Nano-Ribbon Incorporating the Spatial Dispersion Effects
}

\author{
Ping Li, Senior Member, IEEE, Li Jun Jiang, Senior Member, IEEE, and Hakan Bağc1, Senior Member, IEEE
}

\begin{abstract}
It is well known that graphene demonstrates spatial dispersion properties, i.e., its conductivity is nonlocal and a function of spectral wave number (momentum operator) q. In this paper, to account for effects of spatial dispersion on transmission of high speed signals along graphene nanoribbon (GNR) interconnects, a discontinuous Galerkin timedomain (DGTD) algorithm is proposed. The atomically-thick GNR is modeled using a nonlocal transparent surface impedance boundary condition (SIBC) incorporated into the DGTD scheme. Since the conductivity is a complicated function of $q$ (and one cannot find an analytical Fourier transform pair between $q$ and spatial differential operators), an exact time domain SIBC model cannot be derived. To overcome this problem, the conductivity is approximated by its Taylor series in spectral domain under low-q assumption. This approach permits expressing the time domain SIBC in the form of a second-order partial differential equation (PDE) in current density and electric field intensity. To permit easy incorporation of this PDE with the DGTD algorithm, three auxiliary variables, which degenerate the second-order (temporal and spatial) differential operators to first-order ones, are introduced. Regarding to the temporal dispersion effects, the auxiliary differential equation (ADE) method is utilized to eliminates the expensive temporal convolutions. To demonstrate the applicability of the proposed scheme, numerical results, which involve characterization of spatial dispersion effects on the transfer impedance matrix of GNR interconnects, are presented.
\end{abstract}

Index Terms-Auxiliary differential equation (ADE) method, discontinuous Galerkin time-domain (DGTD) method, graphene nano-ribbon (GNR), nonlocal conductivity, spatial dispersion, surface impedance boundary condition (SIBC).

\section{INTRODUCTION}

Two-dimensional (2-D) materials have received significant attention in the last decade due to their remarkable electrical, thermal and mechanic properties features. Graphene is the most well-known and most studied among these materials [1]. Its conductivity can be dynamically tuned via electrostatic gating, magnetostatic gating, or chemical doping, which makes graphene the material of choice for designing novel electromagnetic (EM) and/or electronic devices such as nano-

This work is supported by the National Natural Science Foundation of China (NSFC) under Grant 61701423, and in part by NSFC 61674105, 61622106, 61701424, and in part by UGC of Hong Kong (AoE/P-04/08).

P. Li and L. J. Jiang are with the Department of Electrical and Electronic Engineering, The University of Hong Kong, Hong Kong SAR., China (Corresponding authors: Ping Li (liping@eee.hku.hk) and Li Jun Jiang (jianglj@hku.hk).

H. Bağc1 is with the Division of Computer, Electrical, and Mathematical Sciences and Engineering and the Center for Uncertainty Quantification in Computational Science and Engineering, King Abdullah University of Science and Technology (KAUST), Thuwal, 23955-6900, Saudi Arabia. transistors [2], interconnects [3], reconfigurable terahertz antennas [6], [7], tunable filters [4], and phase shifters [5].

Numerical algorithms, which have been developed in recent years to characterize EM interactions on graphene-based devices include the method of moments (MoM) [8], [9], the finite difference time-domain (FDTD) method [10]-[12], the discontinuous Galerkin time-domain (DGTD) method [13], [14], and the partial element equivalent circuit (PEEC) method [15], [16]. Indeed, these algorithms have been extensively used to investigate transmission, reflection, and absorption of EM fields on graphene sheets as well as generation of graphene surface plasmon polaritons (SPPs) within the gigahertz $(\mathrm{GHz})$ terahertz $(\mathrm{THz})$ frequency band. All these methods model the atomically-thick graphene using an equivalent surface impedance boundary condition (SIBC) to avoid very-fine volumetric discretization of the graphene layer, which would dramatically increase the number of unknowns and decrease the time step size (through Courant-Friedrichs-Lewy (CFL) condition) in the case of explicit time-domain methods. Surface conductivity of the SIBC is either a scalar under electrostatic biasing or a tensor due to quantum hall effect under magnetostatic biasing. In either case, surface conductivity is frequency dependent, i.e. time domain methods have to account for temporal dispersive effects. This can be done using the auxiliary differential equation method (ADE) in [13], [17] or the finite integration technique (FIT) in [14].

On the other hand, none of the methods mentioned above has the capability to account for spatial dispersion that results in nonlocal effects. Indeed, it has been demonstrated that the nonlocality of the graphene's conductivity has a significant influence on the propagation of the surface waves on graphene nano-ribbons (GNRs), especially affecting the near-field confinement, and the propagation constant. Consequently, the effective characteristic impedance of the GNR is dramatically altered [18]-[24]. In [18], the analytical anisotropic Dyadic Green's function method is proposed to handle graphenes over infinitely large substrates while in To analyze a general graphene structure, in [19]-[21], three similar approaches based on the integral equation (IE) method are developed which investigate the dispersion and characteristic impedance of GNRs. As an alternative of full-wave method, the equivalent circuit representation schemes [23]-[25] have been proposed to investigate the effects of the nonlocality with the help of TE and TM transverse resonance method. These methods make use of analytical expressions of the nonlocal conductivity in the spectral domain [18], [22], which are derived using semi- 
classical Boltzmann transport equation (BTE) under either the relaxation-time approximation (RTA) or the Bhatnagar-GrossKrook (BGK) approximation. Equipped with the capability of modeling spatial dispersion, these methods are then used to analyze the phase velocity and attenuation constant of the SPPs generated on GNRs and the crosstalk between coupled lines of GNRs.

So far, methods capable of such analysis have been developed only in frequency domain. This is simply due to the fact that an exact analytical expression for the nonlocal conductivity can not be derived directly in time domain. The conductivity is a complicated function of spectral wave number (or momentum operator) $\mathbf{q}$ and one cannot find an analytical Fourier transform pair between $\mathbf{q}$ and spatial differential operators. Consequently, an exact time domain SIBC model cannot be obtained. To overcome this problem, in this work, a low-q assumption for the (inverse) conductivity is adopted. Under the approximation permits expressing the SIBC in the form of a partial differential equation (PDE) in time and space. This PDE that relates surface current density and electric field intensity is solved together with the Maxwell curl equations. The coupled system of equations is then solved using a time domain discontinuous Galerkin (DGTD) method [28][30]. The DGTD method can be thought as a scheme, which hybridizes the finite volume method (FVM) [26] and the finite element method (FEM) [27]. Like the FVM, the DGTD scheme allows the solution across the interface of neighboring elements to be discontinuous. This provides more flexibility in choosing the order and type of the basis functions (compared to the FEM) and makes the (discretized) spatial operators of the DGTD localized to individual elements. This property, which can also be thought of as an element level domain decomposition scheme, makes DGTD method a powerful scheme to solve multiscale structures without suffering from any ill-conditioning issues. It should be noted here to permit easy incorporation the PDE, which represents the SBIC with the DGTD algorithm, three auxiliary variables, which degenerate the second-order (temporal and spatial) differential operators to first-order ones, are introduced. To take care of the temporal dispersion, the introduction of additional auxiliary variables also eliminates the need to compute costly temporal convolutions.

The resulting DGTD framework proposed in this work to analyze nonlocality/spatial dispersion effects on GNRs is an attractive alternative to the methods mentioned above. The proposed method operates in time domain this means it is more suitable to investigate signal distortion, propagation delay, and jitter and it can be directly used to obtain the eyediagram (indispensable in signal integrity analysis). Moreover, the proposed DGTD scheme provides broadband characterization/data using only a single simulation. To the best of authors' knowledge, this is the first work that facilitates the nonlocal characterization in the time domain using a DGTD framework.

The rest of this paper is organized as follows. Section II provides the details of the formulation underlying the DGTD framework, including the derivation of the PDE representing the SIBC under the low-q assumption, transformation of the second-order PDE into a first-order one, and DGTD-based discretization of the coupled system of the SIBC and Maxwell equations. In Section III, several numerical examples are presented to demonstrate the effects of the nonlocality on the characteristics of GNRs. Finally, Section IV draws conclusions and provides directions for future research.

\section{THEORY AND MATHEMATICAL FORMULATION}

\section{A. Surface Impedance Boundary Condition in the Presence of Spatial Dispersion}

Since the thickness of a graphene layer is much smaller than the graphene's skip depth, the layer can be represented by an equivalent SIBC. The SIBC enforces

$$
\begin{aligned}
& \hat{\mathbf{n}} \times\left(\mathbf{E}^{2}-\mathbf{E}^{1}\right)=0 \\
& \boldsymbol{\sigma}_{g}^{-1} \cdot\left[\hat{\mathbf{n}} \times\left(\mathbf{H}^{2}-\mathbf{H}^{1}\right)\right]=\boldsymbol{\sigma}_{g}^{-1} \cdot \mathbf{J}=\mathbf{E} .
\end{aligned}
$$

Here, $\mathbf{E}^{1}$ and $\mathbf{E}^{2}$ and $\mathbf{H}^{1}$ and $\mathbf{H}^{2}$ are the total electric and magnetic field intensities on the upper and lower sides of the graphene layer, respectively, $\hat{\mathbf{n}}$ is the unit normal vector of the upper side pointing away from the graphene layer, and $\boldsymbol{\sigma}_{g}$ is the graphene's surface conductivity, and $\mathbf{J}$ and $\mathbf{E}$ are the polarization current density and electric field intensity induced inside the graphene layer. It should be noted here that (2) demonstrates that the continuity of tangential component of the magnetic field intensity is disrupted by $\mathbf{J}$. Usually, $\boldsymbol{\sigma}_{g}$ is assumed to be independent of the spectral wave number, thus only spatially-localized model is considered. However, when the dimensions of the graphene ribbon enters are in the nanometer scale [hence the name graphene nano-ribbon $(\mathrm{GNR})], \boldsymbol{\sigma}_{g}$ becomes spatially-dispersive [18]. This means that the interaction between the EM field and the graphene does not depend only on the field at the present position, but is also a function of the fields in the surrounding environment. Consequently, one can use the term nonlocal conductivity [18], [22].

In the spectral domain, the analytical expression of the nonlocal conductivity $\boldsymbol{\sigma}_{g}$ is derived using the semi-classical Boltzmann transport equation under the BGK approximation which explicitly enforces the charge conservation. Based on [20], [22], it is interestingly noted that the nonlocal conductivity tensor in the rectangular coordinates system can be transformed to a diagonal form in a polar coordinate system [22], namely,

$$
\begin{aligned}
\sigma_{g}(q) & =\left[\begin{array}{cc}
\sigma_{\rho}(q) & 0 \\
0 & \sigma_{\phi}(q)
\end{array}\right] \\
& =\sigma_{\phi}(q) \overline{\overline{\mathbf{1}}}+\frac{1}{q^{2}}\left[\sigma_{\rho}(q)-\sigma_{\phi}(q)\right] \mathbf{q q}
\end{aligned}
$$


where

$$
\begin{aligned}
& \sigma_{0}=-\frac{j e^{2} k_{B} T}{\pi \hbar^{2}\left(\omega-j \tau^{-1}\right)}\left[\frac{\mu_{c}}{k_{B} T}+2 \ln \left(\mathrm{e}^{-\mu_{\mathrm{c}} / \mathrm{k}_{\mathrm{B}} \mathrm{T}}+1\right)\right] \\
& \sigma_{\phi}(q)=\frac{2 \sigma_{0}(1-\chi(q))}{\alpha q^{2}}, \quad \chi(q)=\sqrt{\left(1-\alpha q^{2}\right)} \\
& \sigma_{\rho}(q)=\frac{1}{-2 \beta[1-\chi(q)]+\chi(q)} \sigma_{\phi}(q) \\
& \alpha=\frac{V_{F}^{2}}{\left(\omega-j \tau^{-1}\right)^{2}} \\
& \beta=-\frac{j \tau^{-1}}{2 \omega}
\end{aligned}
$$

, and $\mathbf{q}$ is the spectral wave vector, $\omega$ is the frequency, $k_{B}$ is the Boltzmann constant, $T$ is the Kelvin temperature, $\hbar$ is the reduced Planck's constant, $\tau$ is the scattering time, $V_{F}$ is the Fermi velocity, $\mu_{c}$ is the chemical potential, and $e$ is the electronic charge. In this work it is assumed that $T=300 \mathrm{~K}$ and $v_{F} \approx 10^{6} \mathrm{~m} / \mathrm{s}$.

After a lengthy mathematical manipulations, the expression of $\boldsymbol{\sigma}_{g}$ can be written in the following compact form [18], [21], [22]:

$$
\boldsymbol{\sigma}_{g}(q)=\sigma_{\phi}(q)\left[\mathbf{1}+\frac{\psi(q) \mathbf{q q}}{q^{2}}\right]
$$

Here,

$$
\psi(q)=\frac{(1+2 \beta)[1-\chi(q)]}{\chi(q)-2 \beta[1-\chi(q)]}
$$

From (4), the inverse of the conductivity involved in (2) can be readily calculated as [21]

$$
\boldsymbol{\sigma}_{g}^{-1}(q)=\frac{1}{\phi(q)}\left[\mathbf{1}-\frac{\psi(q)}{q^{2}(1+\psi(q))} \mathbf{q q}\right]
$$

In order to model the nonlocal effect by the DGTD method, the spectral domain expression must be converted to the space domain formulation via Fourier transform. Unfortunately, due to the complex relation with the wave number $\mathbf{q}$, the analytical transformation pair is not available. One remedy of this difficulty is resorting to the approximated model. Namely, in the low- $q$ region, the nonlocal conductivity in (5) can be well represented by finite Taylor series. To keep the third-order accuracy, we have [21]

$$
\boldsymbol{\sigma}_{\boldsymbol{g}}^{-\mathbf{1}}(\mathbf{q}) \simeq \frac{1}{\sigma_{0}}\left[\left(1-\frac{\alpha \mathbf{q}^{2}}{4}\right) \mathbf{1}-\frac{\alpha}{2}(1+2 \beta) \mathbf{q q}\right]
$$

By Fourier transform, the spectral wave number (or momentum operator) $\mathbf{q}$ is mapped to a differential operator $\mathbf{q} \rightarrow j \nabla_{t}$ (e.g., $\nabla_{t}=\hat{\mathbf{x}}_{0} \partial_{x}+\hat{\mathbf{y}}_{0} \partial_{y}$ provided that the graphene is placed in the $x y$ plane.), i.e., (6) is correspondingly revised to

$$
\mathbf{J}+\frac{\alpha}{4} \nabla_{t} \nabla_{t} \cdot \mathbf{J}+\alpha\left(\beta+\frac{1}{2}\right) \nabla_{t} \nabla_{t} \cdot \mathbf{J}=\sigma_{0} \mathbf{E}_{t}
$$

To degenerate the second-order spatial differential operator in (7) to first-order one that can be resolved by DGTD algorithm as well as to model the temporal dispersion effects, two scalar auxiliary variables are introduced. Namely,

$$
\begin{aligned}
& Q_{0}=-\frac{v_{F}^{2}}{\left(j \omega+\tau^{-1}\right)} \nabla \cdot \mathbf{J} \\
& Q_{1}=-\frac{\tau^{-1} v_{F}^{2}}{j \omega\left(j \omega+\tau^{-1}\right)} \nabla \cdot \mathbf{J}
\end{aligned}
$$

Via inverse Fourier transform, three time-domain PDEs can be established as:

$$
\begin{aligned}
& \frac{\partial \mathbf{J}}{\partial t}+\tau^{-1} \mathbf{J}+\frac{3}{4} \nabla Q_{0}+\frac{1}{2} \nabla Q_{1}=\sigma_{0} \mathbf{E} \\
& \frac{\partial Q_{0}}{\partial t}+\tau^{-1} Q_{0}+v_{F}^{2} \nabla \cdot \mathbf{J}=0 \\
& \frac{\partial^{2} Q_{1}}{\partial t^{2}}+\tau^{-1} \frac{\partial Q_{1}}{\partial t}+\tau^{-1} v_{F}^{2} \nabla \cdot \mathbf{J}=0
\end{aligned}
$$

To cast (11) into a couple of first-order time-derivative PDEs, another variable $Q_{2}$ defined by

$$
Q_{2}=\frac{\partial Q_{1}}{\partial t}+\tau^{-1} Q_{1}
$$

As a result, (11) is rewritten as

$$
\frac{\partial Q_{2}}{\partial t}+\tau^{-1} v_{F}^{2} \nabla \cdot \mathbf{J}=0
$$

Due to the presence of divergence operator, the nodal basis function is used to expand each component of the polarization current. Thus, we have the separated equations:

$$
\begin{aligned}
& \frac{\partial J_{\xi}}{\partial t}+\tau^{-1} J_{\xi}+\frac{3}{4} \nabla_{\xi} Q_{0}+\frac{1}{2} \nabla_{\xi} Q_{1}=\sigma_{0} \mathbf{E}_{\xi} \\
& \frac{\partial Q_{0}}{\partial t}+\tau^{-1} Q_{0}+v_{F}^{2} \nabla \cdot \mathbf{J}=0 \\
& \frac{\partial Q_{2}}{\partial t}+\tau^{-1} v_{F}^{2} \nabla \cdot \mathbf{J}=0 \\
& \frac{\partial Q_{1}}{\partial t}+\tau^{-1} Q_{1}=Q_{2}
\end{aligned}
$$

where $\xi$ denotes the $x$ and $y$ components of the polarization current $\mathbf{J}$, respectively.

By facilitating the Galerkin testing to the above five equations in the $i$-th graphene patch (In this, the graphene is meshed by triangles):

$$
\begin{aligned}
\int_{\Omega_{i}} \psi_{k}^{i} \frac{\partial J_{\xi}}{\partial} t d \mathbf{r} & =-\tau^{-1} \int_{\Omega_{i}} \psi_{k}^{i} J_{\xi} d \mathbf{r}+\frac{3}{4} \int_{\Omega_{i}} Q_{0} \nabla_{\xi} \psi_{k}^{i} d \mathbf{r}+ \\
& \frac{1}{2} \int_{\Omega_{i}} Q_{1} \nabla_{\xi} \psi_{k}^{i} d \mathbf{r}+\sigma_{0} \int_{\Omega_{i}} \psi_{k}^{i} E_{\xi} d \mathbf{r}- \\
& \frac{3}{4} \int_{l_{i}} \phi_{k}^{i}\left[\hat{n}_{\xi}^{i} Q_{0}^{*}\right] d l-\frac{1}{2} \int_{l_{i}} \phi_{k}^{i}\left[\hat{n}_{\xi}^{i} Q_{1}^{*}\right] d l
\end{aligned}
$$

$$
\begin{aligned}
\int_{\Omega_{i}} \psi_{k}^{i} \frac{\partial Q_{0}}{\partial t} d \mathbf{r}= & -\tau^{-1} \int_{\Omega_{i}} \psi_{k}^{i} Q_{0} d \mathbf{r}+v_{F}^{2} \int_{\Omega_{i}} \mathbf{J} \cdot \nabla \psi_{k}^{i} d \mathbf{r}- \\
& v_{F}^{2} \int_{l_{i}} \phi_{k}^{i}\left[\hat{\mathbf{n}}^{i} \cdot \mathbf{J}^{*}\right] d l
\end{aligned}
$$$$
\int_{\Omega_{i}} \psi_{k}^{i} \frac{\partial Q_{2}}{\partial t} d \mathbf{r}=\frac{\tau}{v_{F}^{2}} \int_{\Omega_{i}} \mathbf{J} \cdot \nabla \psi_{k}^{i} d \mathbf{r}-\frac{\tau}{v_{F}^{2}} \int_{l_{i}} \phi_{k}^{i}\left[\hat{\mathbf{n}}^{i} \cdot \mathbf{J}^{*}\right] d l
$$

$$
\int_{\Omega_{i}} \psi_{k}^{i} \frac{\partial Q_{1}}{\partial t} d \mathbf{r}=-\tau^{-1} \int_{\Omega_{i}} \psi_{k}^{i} Q_{1} d \mathbf{r}+\int_{\Omega_{i}} \psi_{k}^{i} Q_{2} d \mathbf{r}
$$




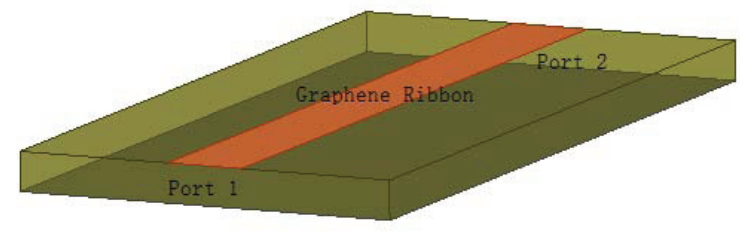

Fig. 1. The schematic illustration of the graphene nano-ribbon transmission line placed over a substrate.

where $\psi_{k}^{i}$ denotes the $k$-th scalar test basis function, $\hat{\mathbf{n}}^{i}$ represents the outward normal vector pertinent to the corresponding triangle edge.

Since the solutions in DGTD method are allowed to be discontinuous at the interface of neighboring domains, the values with superscript ${ }^{*}$ from (19) to (23) are named as numerical flux that are used for communication between adjacent domains in order to guarantee an unique solution. In this work, the definition of the numerical fluxes are given as [31]

$$
\begin{aligned}
& \hat{\mathbf{n}}^{i} \cdot \mathbf{J}^{*}=C_{10}\left(\hat{\mathbf{n}}^{i} \cdot \mathbf{J}^{i}+\hat{\mathbf{n}}^{i} \cdot \mathbf{J}^{j}\right)+C_{11}\left(\hat{\mathbf{n}}^{i} \cdot \mathbf{J}^{i}-\hat{\mathbf{n}}^{i} \cdot \mathbf{J}^{j}\right) \\
& +C_{12}\left(Q^{i}-Q^{j}\right) \\
& Q^{*}=C_{20}\left(Q^{i}+Q^{j}\right)+C_{21}\left(\hat{\mathbf{n}}^{i} \cdot \mathbf{J}^{i}-\hat{\mathbf{n}}^{i} \cdot \mathbf{J}^{j}\right) \\
& +C_{22}\left(Q^{i}-Q^{j}\right)
\end{aligned}
$$

In this work, the the coefficients are set as $C_{10}=0.5, C_{11}=0$, $C_{12}=-4, C_{20}=0.5, C_{21}=0$, and $C_{22}=0$.

By approximating the unknowns via nodal basis functions: $J_{\xi}=\sum_{l=1}^{n_{J_{\xi}}^{i}} j_{\xi, l}^{i}(t) \psi_{l}^{i}(\mathbf{r})$ with $\xi=x, y$, and $Q_{k}=$ $\sum_{m=1}^{n_{Q_{k}}^{i}} q_{k, m}^{i}(t) \psi_{m}^{i}(\mathbf{r})$ with $k=0,1,2$, in which $n_{J_{\xi}}$ and $n_{Q_{k}}$ denotes the number of nodal basis functions in each mesh cell corresponding to $J_{\xi}$ and $Q_{k}$, respectively. Then, (19) to (22) will be transformed to the following semi-discrete matrix equations as

$$
\begin{aligned}
& \overline{\mathbf{M}}_{\xi}^{i} \frac{\partial \mathbf{j}_{\xi}^{i}}{\partial t}=-\tau^{-1} \overline{\mathbf{M}}_{\xi}^{i} \mathbf{j}_{\xi}^{i}+\overline{\mathbf{S}}_{\xi, 0}^{i} \mathbf{q}_{0}^{i}+\overline{\mathbf{S}}_{\xi, 1}^{i} \mathbf{q}_{1}^{i}+\overline{\mathbf{F}}_{\xi, 0}^{i i} \mathbf{q}_{0}^{i}+ \\
& \overline{\mathbf{F}}_{\xi, 0}^{i j} \mathbf{q}_{0}^{j}+\overline{\mathbf{F}}_{\xi, 1}^{i i} \mathbf{q}_{1}^{i}+\overline{\mathbf{F}}_{\xi, 1}^{i j} \mathbf{q}_{1}^{j} \\
& \overline{\mathbf{M}}_{Q_{0}}^{i} \frac{\partial \mathbf{q}_{0}^{i}}{\partial t}=-\tau^{-1} \overline{\mathbf{M}}_{Q_{0}}^{i} \mathbf{q}_{0}^{i}+\overline{\mathbf{S}}_{0 x}^{i} \mathbf{j}_{x}^{i}+\overline{\mathbf{S}}_{0 y}^{i} \mathbf{j}_{y}^{i}+\overline{\mathbf{F}}_{0 x}^{i i} \mathbf{j}_{x}^{i}+ \\
& \overline{\mathbf{F}}_{0 x}^{i j} \mathbf{j}_{x}^{j}+\overline{\mathbf{F}}_{0 y}^{i i} \mathbf{j}_{y}^{i}+\overline{\mathbf{F}}_{0 y}^{i j} \mathbf{j}_{y}^{j} \\
& \overline{\mathbf{M}}_{Q_{2}}^{i} \frac{\partial \mathbf{q}_{2}^{i}}{\partial t}=\overline{\mathbf{S}}_{2 x}^{i} \mathbf{j}_{x}^{i}+\overline{\mathbf{S}}_{2 y}^{i} \mathbf{j}_{y}^{i}+\overline{\mathbf{F}}_{2 x}^{i i} \mathbf{j}_{x}^{i}+\overline{\mathbf{F}}_{2 x}^{i j} \mathbf{j}_{x}^{j}+ \\
& \overline{\mathbf{F}}_{2 y}^{i i} \mathbf{j}_{y}^{i}+\overline{\mathbf{F}}_{2 y}^{i j} \mathbf{j}_{y}^{j} \\
& \overline{\mathbf{M}}_{Q_{1}}^{i} \frac{\partial \mathbf{q}_{1}^{i}}{\partial t}=-\tau^{-1} \overline{\mathbf{M}}_{Q_{1}}^{i} \mathbf{q}_{1}^{i}+\overline{\mathbf{M}}_{Q_{2}}^{i} \mathbf{q}_{2}^{i}
\end{aligned}
$$

where $\overline{\mathbf{M}}^{i}, \overline{\mathbf{S}}^{i}$, and $\overline{\mathbf{F}}$ represent mass, stiffness, and flux matrices, respectively; $\mathbf{j}$ and $\mathbf{q}$ are column vectors containing the unknown expansion coefficients; $\xi$ represents the $x$ and $y$ components of $\mathbf{J}$, respectively.

\section{B. DGTD Formulation of Maxwell's Equations}

To solve the computational domain of interest, we firstly split the whole region $\Omega$ into into $N$ non-overlapping tetrahedrons $\Omega_{i}$ with boundary $\partial \Omega_{i}$. In element $i$, the electric

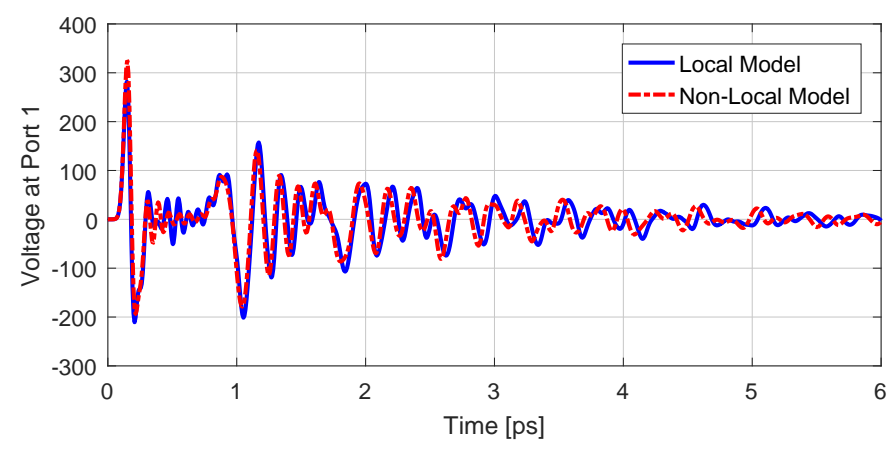

(a) Voltage at Port 1

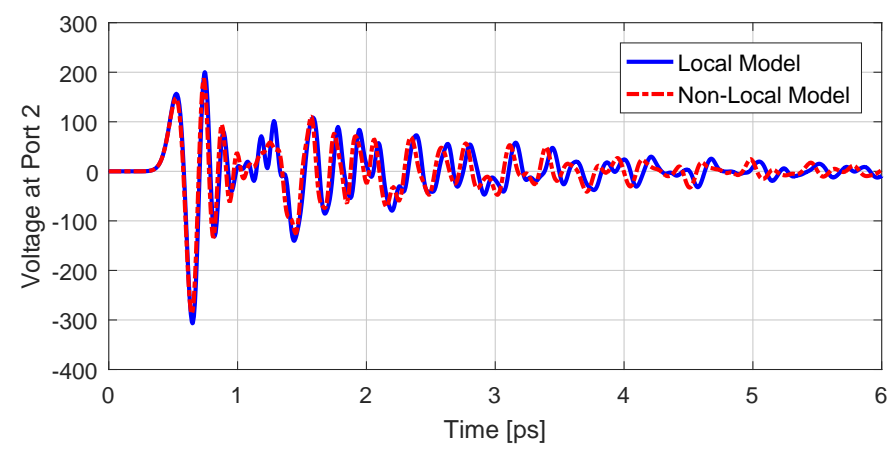

(b) Voltage at Port 2

Fig. 2. The recorded temporal voltage at Port 1 and Port 2 of the nano-ribbon transmission line using either local or non-local conductivity model.

field $\mathbf{E}$ and magnetic field $\mathbf{H}$ are approximated by 3-D vector basis functions $\Phi^{i}(\mathbf{r}), \Psi^{i}(\mathbf{r})$ [28]: $\mathbf{E}^{i}=\sum_{k=1}^{n_{e}^{i}} e_{k}^{i}(t) \boldsymbol{\Phi}_{k}^{i}(\mathbf{r})$ and $\mathbf{H}^{i}=\sum_{l=1}^{n_{h}^{i}} h_{k}^{i}(t) \mathbf{\Psi}_{l}^{i}(\mathbf{r})$, in which $n_{e}^{i}$ and $n_{h}^{i}$ are the number of vector basis functions for $\mathbf{E}$ and $\mathbf{H}$ in $i$-th element, respectively; $e_{k}^{i}$ and $h_{k}^{i}$ are the unknown time-dependent coefficients of basis functions $\Phi^{i}(\mathbf{r})$ and $\Psi^{i}(\mathbf{r})$, respectively.

Apply the DG test over the two first-order time-derivative Maxwell's curl equations, we can obtain

$$
\begin{aligned}
& \int_{\Omega_{i}} \boldsymbol{\Phi}_{k}^{i} \cdot\left[\epsilon_{i} \frac{\partial \mathbf{E}^{i}}{\partial t}-\nabla \times \mathbf{H}^{i}\right] d \mathbf{r}= \\
& \sum_{f=1}^{4} \int_{\partial \Omega_{i, f}} \boldsymbol{\Phi}_{k}^{i} \cdot\left[\hat{\mathbf{n}}_{i, f} \times\left(\mathbf{H}_{f}^{*}-\mathbf{H}^{i}\right)\right] d \mathbf{r} \\
& \int_{\Omega_{i}} \mathbf{\Psi}_{l}^{i} \cdot\left[\mu_{i} \frac{\partial \mathbf{H}^{i}}{\partial t}+\nabla \times \mathbf{E}^{i}\right] d \mathbf{r}= \\
& \sum_{f=1}^{4} \int_{\partial \Omega_{i, f}} \mathbf{\Psi}_{l}^{i} \cdot\left[\hat{\mathbf{n}}_{i, f} \times\left(\mathbf{E}^{i}-\mathbf{E}_{f}^{*}\right)\right] d \mathbf{r}
\end{aligned}
$$

where $\hat{\mathbf{n}}_{f}^{i} \times \mathbf{E}_{f}^{*}$ and $\hat{\mathbf{n}}_{f}^{i} \times \mathbf{H}_{f}^{*}$ are numerical fluxes used for information exchange between neighboring elements. In order to consider the presence of graphene, the SIBC in (2) is incorporated by re-formulating the numerical fluxes on the basis of the Rankine-Hugoniot Jump Relations [13], [14], [26]. 


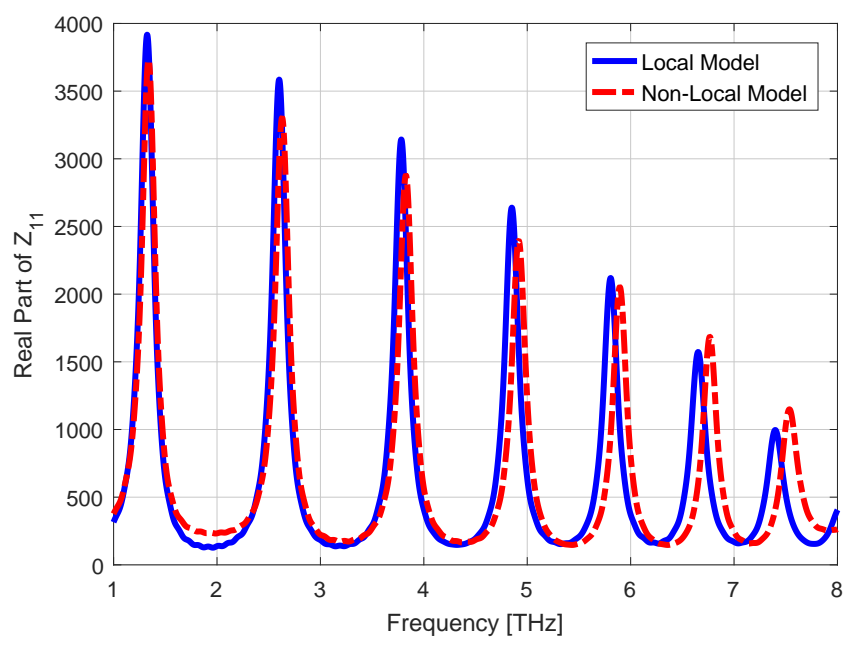

(a) Real part of $\mathrm{Z}_{11}$

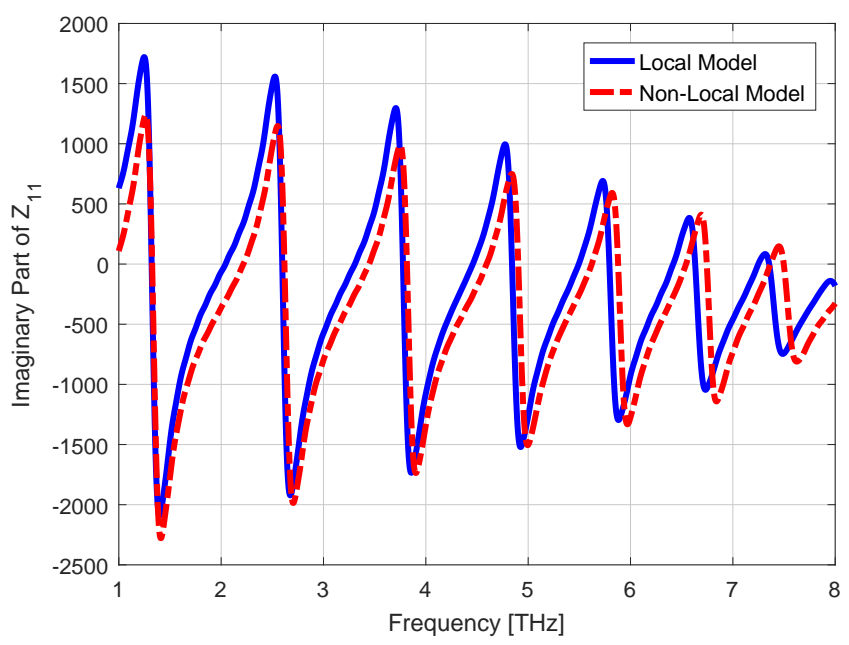

(b) Imaginary part of $\mathrm{Z}_{11}$

Fig. 3. The calculated impedance matrix elements $\mathrm{Z}_{11}$ with regarding the presence of spatial dispersion effects for the nano-ribbon transmission line. For comparison, its counterpart involving only local effects is also presented.

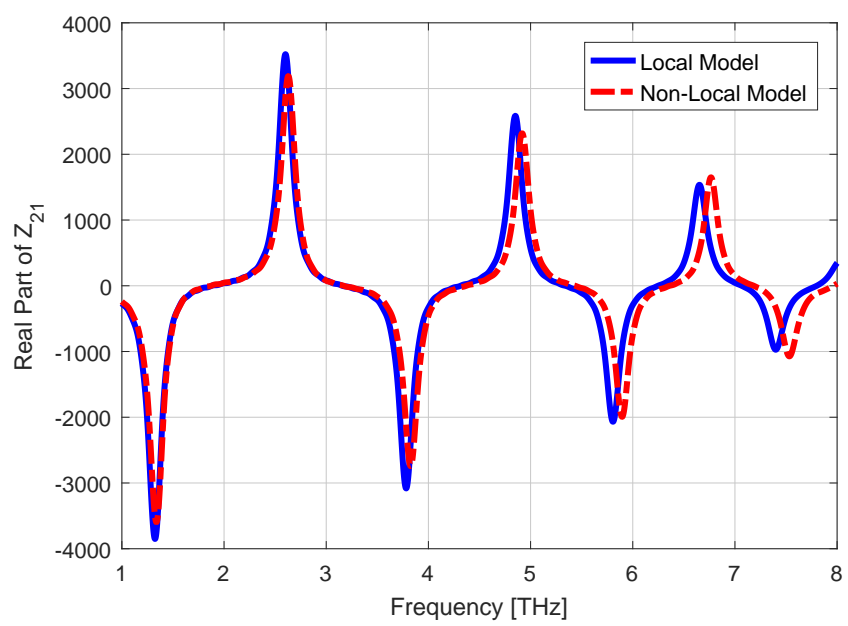

(a) Real part of $\mathrm{Z}_{21}$

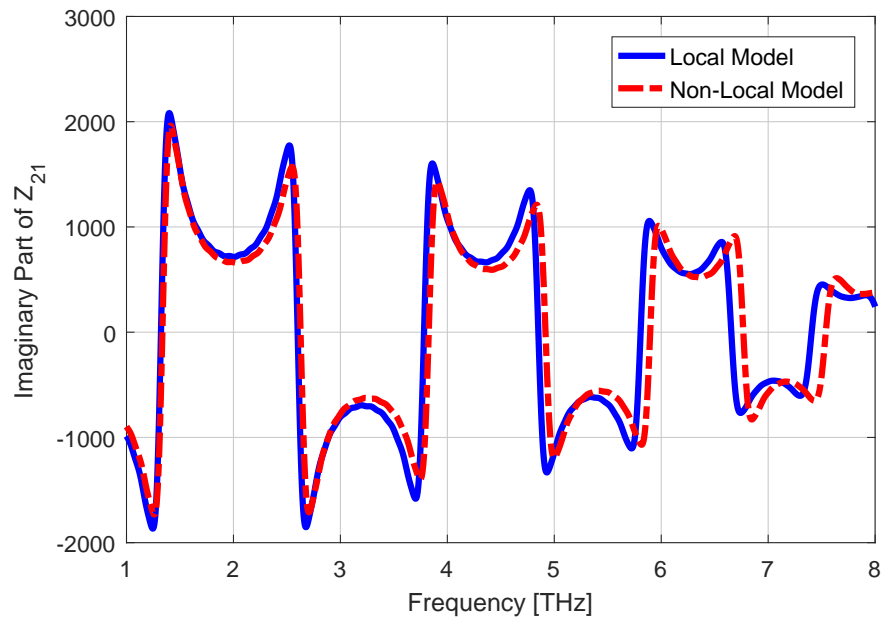

(b) Imaginary part of $Z_{21}$

Fig. 4. The calculated impedance matrix elements $Z_{21}$ with regarding the presence of spatial dispersion effects for the nano-ribbon transmission line. For comparison, its counterpart involving only local effects is also presented.

That is,

$\hat{\mathbf{n}}_{i, f} \times \mathbf{H}_{f}^{*}=\hat{\mathbf{n}}_{i, f} \times\left[\frac{\left(Z^{i} \mathbf{H}^{i}+Z_{f}^{j} \mathbf{H}_{f}^{j}\right)+\hat{\mathbf{n}}_{i, f} \times\left(\mathbf{E}^{i}-\mathbf{E}_{f}^{j}\right)}{Z^{i}+Z_{f}^{j}}\right.$

$$
\left.+\alpha_{g} \frac{Z^{j} \mathbf{J}_{g}^{i}}{\left(Z^{i}+Z_{f}^{j}\right)}\right]
$$

$\hat{\mathbf{n}}_{i, f} \times \mathbf{E}_{f}^{*}=\hat{\mathbf{n}}_{i, f} \times\left[\frac{\left(Y^{i} \mathbf{E}^{i}+Y_{f}^{j} \mathbf{E}_{f}^{j}\right)+\hat{\mathbf{n}}_{i, f} \times\left(\mathbf{H}_{f}^{j}-\mathbf{H}^{i}\right)}{Y^{i}+Y_{f}^{j}}\right.$

$$
\left.-\alpha_{g} \frac{\mathbf{J}_{g}^{i}}{2\left(Y^{i}+Y_{f}^{j}\right)}\right]
$$

where $Z^{i}=\sqrt{\mu^{i} / \epsilon^{i}}$ and $Z_{f}^{j}=\sqrt{\mu_{f}^{j} / \epsilon_{f}^{j}}$ represent the characteristic impedance of element $i$ and the neighboring at the $f$-th face, respectively.

In (30) and (31), the parameter $\alpha_{g}$ is given by

$$
\alpha_{g}=\left\{\begin{array}{l}
1, f=f_{g} \\
0, f \neq f_{g}
\end{array}\right.
$$

with $f_{g}$ denoting the face over the graphene.

With equations from (28) to (31), two semi-discrete matrices can be obtained as

$$
\begin{aligned}
\overline{\mathbf{M}}_{e}^{i} \frac{\partial \mathbf{e}^{i}}{\partial t}=\overline{\mathbf{S}}_{e}^{i} \mathbf{h}^{i} & +\sum_{f=1}^{4}\left(\overline{\mathbf{F}}_{e e}^{i i, f} \mathbf{e}_{f}^{i}+\overline{\mathbf{F}}_{e e}^{i j, f} \mathbf{e}_{f}^{j}\right. \\
& \left.+\overline{\mathbf{F}}_{e h}^{i i, f} \mathbf{h}_{f}^{i}+\overline{\mathbf{F}}_{e h}^{i j, f} \mathbf{h}_{f}^{j}\right)+\alpha_{g} \overline{\mathbf{F}}_{e c}^{i i, f_{g}} \mathbf{c}^{i}
\end{aligned}
$$




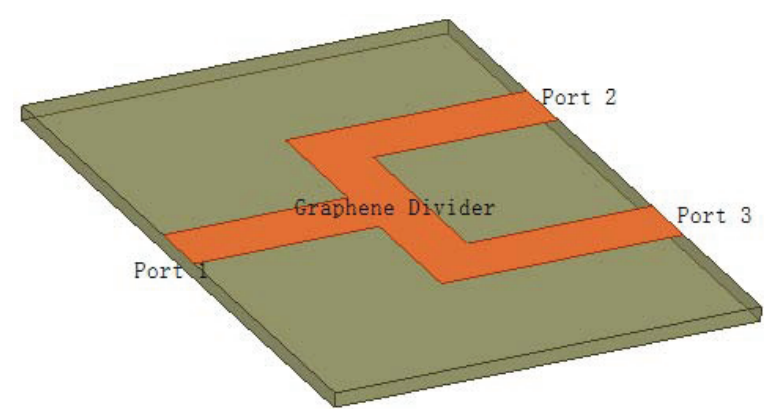

Fig. 7. The schematic illustration of the graphene nano-divider placed over a substrate.

$$
\begin{aligned}
\overline{\mathbf{M}}_{h}^{i} \frac{\partial \mathbf{h}^{i}}{\partial t}=-\overline{\mathbf{S}}_{h}^{i} \mathbf{e}^{i} & +\sum_{f=1}^{4}\left(\overline{\mathbf{F}}_{h h}^{i i, f} \mathbf{h}_{f}^{i}+\overline{\mathbf{F}}_{h h}^{i j, f} \mathbf{h}_{f}^{j}\right. \\
& \left.+\overline{\mathbf{F}}_{h e}^{i i, f} \mathbf{e}_{f}^{i}+\overline{\mathbf{F}}_{h e}^{i j, f} \mathbf{e}_{f}^{j}\right)+\alpha_{g} \overline{\mathbf{F}}_{h e}^{i i, f_{g}} \mathbf{c}^{i}
\end{aligned}
$$

where $\overline{\mathbf{M}}_{e, h}^{i}, \overline{\mathbf{S}}_{e, h}^{i}, \overline{\mathbf{F}}_{e e}, \overline{\mathbf{F}}_{e h}, \overline{\mathbf{F}}_{h e}$, and $\overline{\mathbf{F}}_{h h}$ denote the mass, stiffness and flux matrices, respectively. The detailed definitions can be found in our previous work [13], [14].

To solve the semi-discrete matrix equations from (25) to (27) and together with (33) and (34), the explicit fourstage Runge-Kutta (RK) time-marching method [32], [33] is employed. To ensure stability, the maximum time step size $\delta t$ is determined in terms of the following condition [13]:

$$
c_{0} \delta t \leq \min \left\{l_{\min } \sqrt{\epsilon_{r} \mu_{r}} / 4(p+1)^{2}\right\}
$$

where $c_{0}$ is the free-space light speed, $p$ is the order of basis function, $l_{\min }$ is the minimum edge length.

\section{NumERICAL RESUlts}

In this section, effects of spatial dispersion (i.e. nonlocal conductivity) on the characteristics of GNRs are demonstrated via application of the proposed DGTD framework to the analysis of EM field interactions on two different GNRs. The results are compared to those obtained by the same DGTD framework using the local conductivity model.

\section{A. A Graphene Nano-Ribbon Transmission Line}

For the first example, EM field interactions on a GNR, which is located over a silicon substrate, are analyzed using the proposed DGTD framework. The width and length of the GNR are $0.3 \mu \mathrm{m}$ and $3 \mu \mathrm{m}$, respectively; the height of the substrate is $0.05 \mu \mathrm{m}$ and its relative permittivity is 4.0 , as shown in Fig. 1. A current source is applied at Port 1. Two simulations are carried out, one with the nonlocal conductivity model and the other with the local conductivity model. For this simulation, the number of meshes is 23,310 , and 326 triangles are required for the graphene discretization, the total unknowns are 564,330 , the time-step size obtained according to (35) is $1.05 \times 10^{-5} \mathrm{ps}$, and the average CPU time for each step is $0.0548 \mathrm{~s}$. Fig. 2 compares the transient voltages recorded at Port 1 and Port 2 during these simulations. Once the simulations are completed, results are converted into frequency domain and impedance matrix elements $Z_{11}$ and $Z_{21}$ are extracted. Fig. 3 and 4 plot $Z_{11}$ and $Z_{21}$ versus frequency. Both

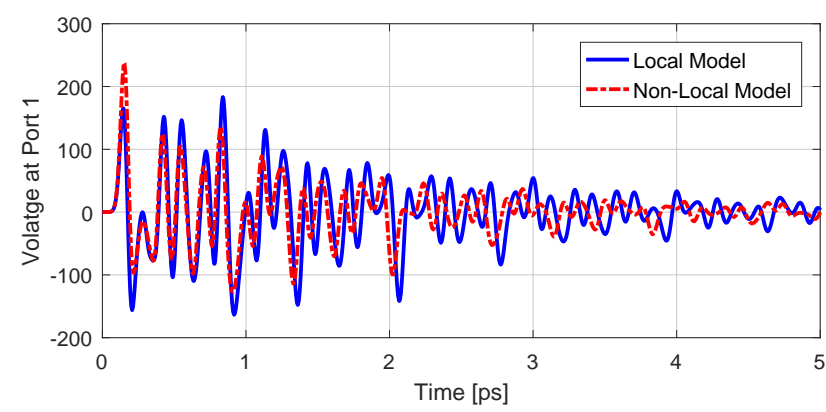

(a) Voltage at Port 1

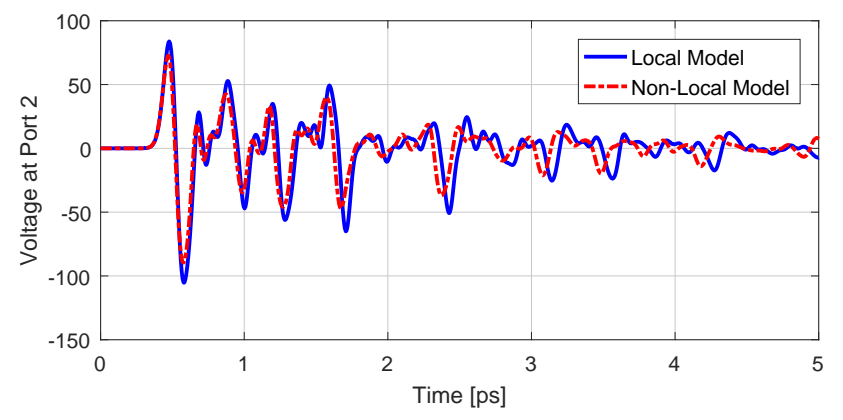

(b) Voltage at Port 2

Fig. 8. The recorded temporal voltage at Port 1 and Port 2 of the nano-power divider using either local or non-local conductivity model.

time and frequency domain results show obvious differences, especially towards the high end of the terahertz band, and demonstrate the importance of using a nonlocal model for the conductivity of the graphene at these frequencies.

The previous study [18], [20], [22], [25] has already shown that the strength of the nonlocal effects will degrade if the width of the graphene ribbon was increased or lower substrate permittivity was employed. To verify this statement, we increase the width of the graphene nano-ribbon to $3 \mu \mathrm{m}$ and simultaneously reduce the relative permittivity of the substrate to 2.2. For this simulation, the required maximum time step size is $7.76 \times 10^{-5}$ ps. In Fig. 5, the voltages at the excitation Port 1 and receiving Port 2 are given. For the purpose of comparison, the results corresponding to local model are also provided. It is interesting to note that the impact of spatial dispersion becomes ignorable, which is consistent with the argument claimed in previous studies. Next, to investigate the impact of the substrate on the nonlocal effects, the width of the GNR is unchanged while the relative permittivity of the substrate is increased to 11.9 , as a result, the time step size is changed to $1.81 \times 10^{-5}$ ps. in Fig. 6 , the calculated port voltages are presented. It is interestingly noted that the nonlocal effect rises. The physics behind these phenomenons are attributed to two folds: (i) When mean free path of the carriers becomes shorter than the dimension of the nano-structure (width in this example), the spatial dispersion becomes negligible; (ii) A high permittivity substrate strengthens the non-locality, thereby a low permittivity substrate effectively mitigates the spatial dispersion effects. Thus, the strength of the nonlocal effect is both dependent on the width and the permittivity of the dielectric substrate. To make sure the validation of low- $q$ approximation, it is more safer if the width of the 


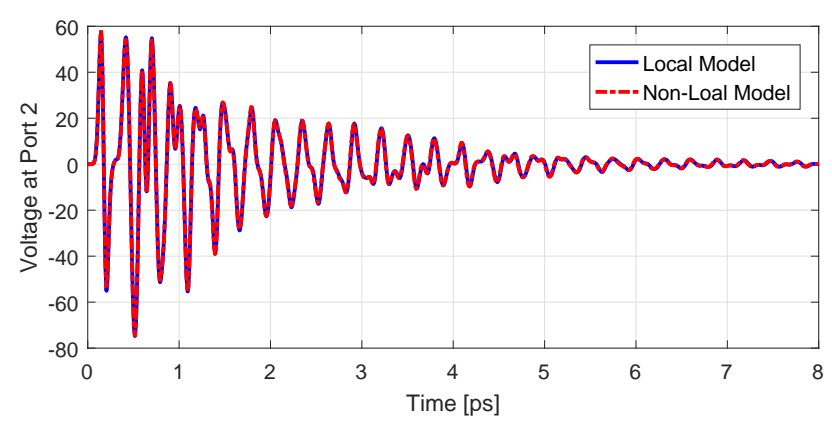

(a) Voltage at Port 1

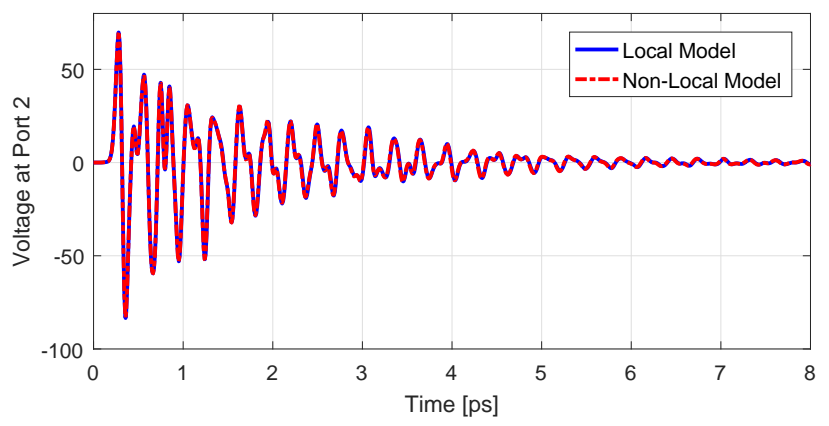

(b) Voltage at Port 2

Fig. 5. The recorded temporal voltage at Port 1 and Port 2 of the new nano-ribbon transmission line using either local or non-local conductivity model.

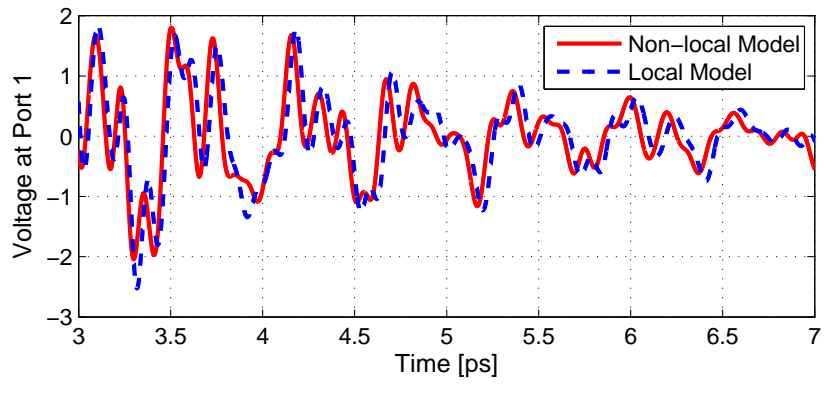

(a) Voltage at Port 1

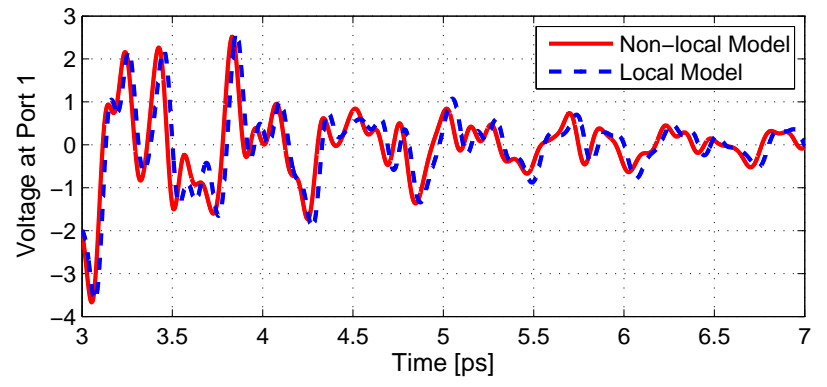

(b) Voltage at Port 2

Fig. 6. The calculated voltage pertinent to the relative permittivity of substrate equal to 11.9.

GNR is no smaller tha $1 \mu \mathrm{m}$ based on our and the previous investigations [20].

\section{B. A Graphene Nano-Ribbon Power Divider}

For the second example, a passive graphene nanocomponent named power divider is investigated, the width of the GNR is $0.2 \mu \mathrm{m}$, and the length of the input stub and the two branches are $0.65 \mu \mathrm{m}$ and $0.85 \mu \mathrm{m}$, respectively; the height of the substrate is $0.05 \mu \mathrm{m}$, the details of the configuration can be found in Fig. 7. For this example, the number of tetrahedron elements is 60,866 and 374 triangles are used to approximate the graphene divider, the corresponding total number of unknowns is $1,454,624$, the resultant time step size is $5.58 \times 10^{-6} \mathrm{ps}$, and CPU time for each time step is around 0.152 s.A current source is applied at Port 1 , and the voltage at Port 1 and 2 are recorded and shown in Fig. 8. For comparison, the results pertinent to the local mode are also presented. Correspondingly, the impedance matrix elements $Z_{11}$ and $Z_{21}$ are evaluated based on the acquired Port voltage and simultaneously compared with the results using local model only. As shown in Fig. 9 and 10, it is noted that the both the real and imaginary parts of the impedance matrix shows a frequency shift and some amplitude variation while the shapes kept similar, which is due to that the nonlocal effects has directly influence on the propagating wave number including the phase constant [19]-[21], thus the relative electric length of the GNR is changed compared with the local model case, which directly results in the variations of the peaks and nulls of the impedance matrix.

\section{CONCLUSION}

A DGTD framework is developed to study effects of the spatial dispersion due to the nonlocality of the graphene's conductivity on the GNR characteristics. The DGTD framework models the graphene layer as an SIBC. The time-space PDE representing this SIBC is obtained from the spectraldomain expression of the graphene's conductivity under low-q assumption. Auxiliary variables are introduced to degenerate this second-order PDE into first-order ones. These auxiliary variables also eliminate the need to compute costly temporal convolutions. To demonstrate the applicability of the DGTD framework, numerical results, which involve characterization of spatial dispersion effects on the transfer impedance matrix of GNR interconnects, are presented, which further verify that the nonlocal effects of GNR is dependent on both the width of GNR and the dielectric substrate.

\section{REFERENCES}

[1] P. Sharma, J. P. Carrier, C. Moldovan, and A. M. Ionescu, "Electromagnetic performance of RF NEMS graphene capacitive switches," IEEE Trans. Nanotechn., vol. 13, no. 1, pp. 70-79, Jan. 2014.

[2] F. schwierz, "Graphene transistors," Nat. Nanotechn., vol. 5, pp. 487-496, Jul. 2010.

[3] C. Xu, H. Li, .and K. Banerjee, "Modeling, analysis, and design of graphene nano-ribbon interconnects," IEEE Trans. Electron Devices, vol. 56, no. 8, pp. 1567-1578, Aug. 2009.

[4] D. C.-Serrano, J. S. G.-Diaz, J. P.-Carrier, and A. A.-Melcon, "Graphenebased plasmonic tunable low-pass filters in the terahertz band," IEEE Trans. Nanotechn., vol. 13, no. 6, pp. 1145-1153, Nov. 2014.

[5] P. Y. Chen, C. Argyropoulos,.and A. Alu, "Terahertz antenna phase shifters using integrally-gated graphene transmission-lines," IEEE Trans. Antennas Propag., vol. 61, no. 4, pp. 1528-1537, Apr. 2013.

[6] M. E. Morote, J. S. G.-Diaz, and J. P.-Carrier, "Sinusoidally modulated graphene leaky-wave antenna for electronic beamscanning at THz," IEEE Trans. Terahertz Sci. Techn., vol. 4, no. 1, pp. 116-122, Jan. 2014. 


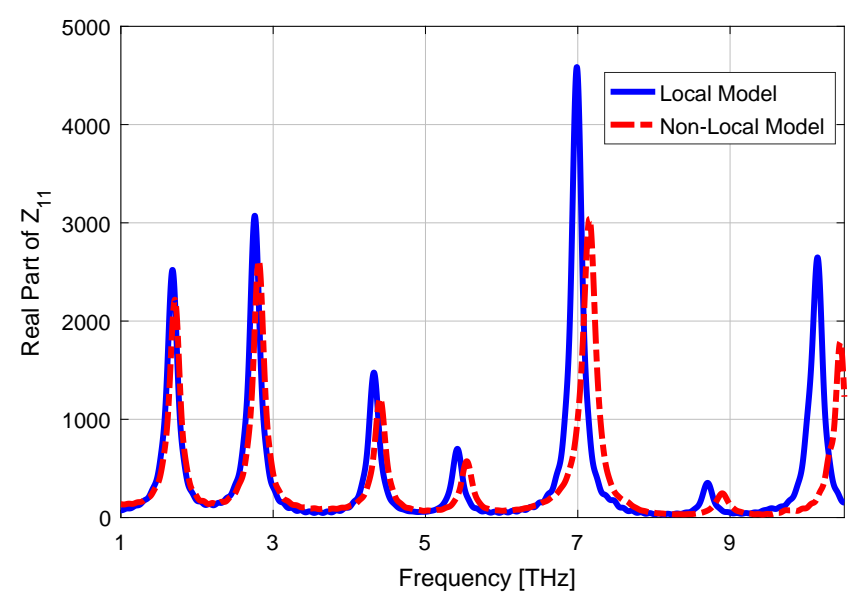

(a) Real part of $\mathrm{Z}_{11}$

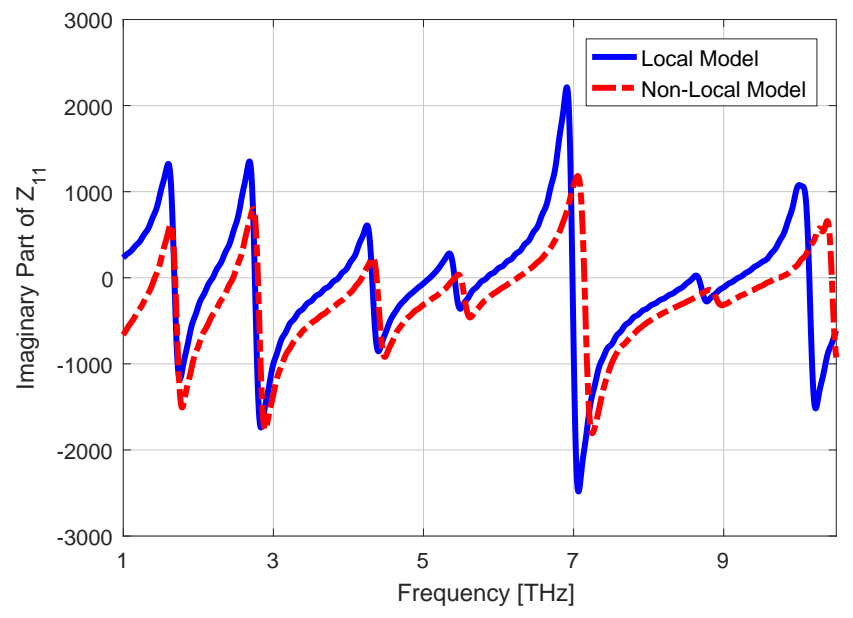

(b) Imaginary part of $Z_{11}$

Fig. 9. The calculated impedance matrix elements $Z_{11}$ with regarding the presence of spatial dispersion effects for the nano-power divider. For comparison, its counterpart involving only local effects is also presented.

[7] D. C.-Serrano and J. S. Gomez-Diaz, "Graphene-based antennas for Terahertz systems: a review," Forum for Electromagn. Res. Methods and Application Techn.

[8] G. W. Hanson, "Dyadic Green's functions for an anisotropic, non-Local model of biased graphene," IEEE Trans. Antennas Propag., vol. 56, no. 3, pp. 747-757, Mar. 2008

[9] O. V. Shapoval, J. S. G-Diaz, J. P-Carrier, J. R. Mosig, and A. I. Nosich, "Integral equation analysis of plane wave scattering by coplanar graphenestrip gratings in the THz range," IEEE Trans. Terahertz Sci. Techn., vol. 6, no. 3, pp. 666-674, Sept. 2013.

[10] H. Lin, M. F. Pantoja, L. D. Angulo, J. Alvarez, R. G. Martin, and S. G. Garcia, "FDTD modeling of graphene devices using complex conjugate dispersion material model," IEEE Micorw. Wireless Compon. Lett., vol. 22, no. 12, pp. 612-614, Dec. 2012.

[11] V. Nayyeri, M. Soleimani, and M. Ramahi, "Wideband modeling of graphene using the finite-difference time-domain metod," IEEE Trans. Antennas Propag., vol. 6, no. 12, pp. 6107-6114, Dec. 2013.

[12] V. Nayyeri, M. Soleimani, and M. Ramahi, "Modeling graphene in the finite-difference time-domain method using a surface boundary condition," IEEE Trans. Antennas Propag., vol. 6, no. 8, pp. 4176-4182, Aug. 2013.

[13] P. Li and L. J. Jiang, "Modeling of magnetized graphene from microwave to THz range by DGTD with a scalar RBC and an ADE," IEEE Trans. Antennas Propag., vol. 63, no. 10, pp. 4458-4467, Oct. 2015.

[14] P. Li, L. J. Jiang, and H. Bagci, "A resistive boundary condition enhanced DGTD scheme for the transient analysis of graphene," IEEE Trans. Antennas Propag., vol. 63, no. 7, pp.3065-3076, Jul. 2015.

[15] Y. Cao, P. Li, L. J. Jiang, and A. E. Rueli, "The derived equivalent circuit

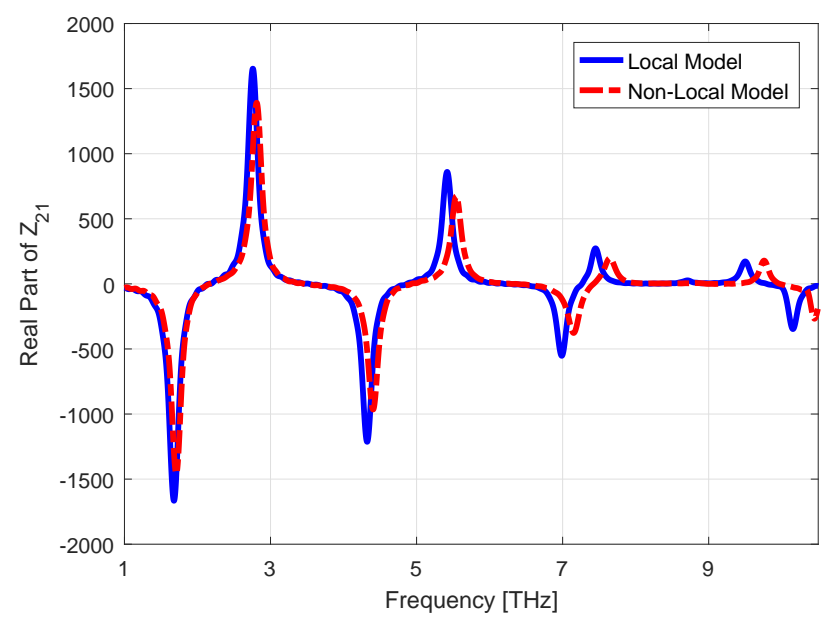

(a) Real part of $Z_{21}$

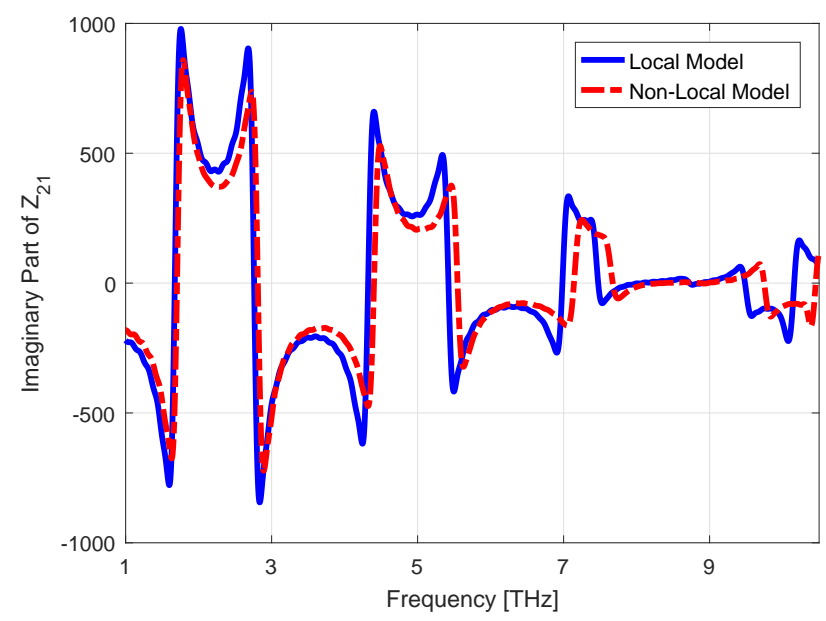

(b) Imaginary part of $Z_{21}$

Fig. 10. The calculated impedance matrix elements $Z_{21}$ with regarding the presence of spatial dispersion effects for nano-power divider. For comparison, its counterpart involving only local effects is also presented.

model for magnetized anisotropic grapheme," IEEE Trans. Antennas and Propag., vol. 65, no. 2, pp. 948-953, Feb. 2017.

[16] D. Romano and G. Antonini, "Partial Element Equivalent CircuitBased Transient Analysis of Graphene-Based Interconnects," IEEE Trans. Electromagn. Compat., vol. 58, no. 3, pp. 801-810, Jun. 2016.

[17] I. Ahmed, E. H. Khoo, and E. Li, "Efficient modeling and simulation of graphene devices with the LOD-FDTD method," IEEE Microw. Wireless Compon. Lett., vol. 23, no. 6, pp. 306-308, Jun. 2013.

[18] G. W. Hanson, "Dyadic Greens functions for an anisotropic, non-local model of biased graphene," IEEE Trans. Antennas Propag., vol. 56, no. 3, pp. 747-757, Mar. 2008.

[19] A. Fallahi, T.Low, M. Tamagnone, and J. P.-Carrier, "Nonlocal electromagnetic response of graphene nanostructures," Phy. Rev. B, 121405(1)(5), 2015.

[20] G. Lovat, R. Araneo, P.Burghignoli, and G. W. Hanson, "Nonlocal effects on surface plasmon polariton propagation in graphene nanoribbons," IEEE Trans. Terahertz Sci. Techn., vol. 5, no. 6, pp. 941-950, Oct. 2015.

[21] R. Araneo, P. Burghignoli, G. Lovat, and G. W. Hanson, "Modal propagation and crosstalk analysis in coupled graphene nanoribbons," IEEE Trans. Electromagn. Compat., vol. 57, no. 4, pp. 726-733, Aug. 2015.

[22] G. Lovat, G. W. Hanson,2, R. Araneo, and P. Burghignoli, "Semiclassical spatially dispersive intraband conductivity tensor and quantum capacitance of graphene," Phy. Rev. B, 115429(1)-(11), 2013.

[23] G. Lovat, P.Burghignoli, and R. Araneo, "Low-frequency dominantmode propagation in spatially dispersive graphene nanowaveguides," IEEE Trans. Electromagn. Compat., vol. 55, no. 2, pp. 328-333, Apri. 2013. 
[24] J. S. G.-Diaz, J. R. Mosig, and J. P.-Carrier, "Effect of spatial dispersion on surface waves propagating along graphene sheets," IEEE Trans. Antennas Propag., vol. 61, no. 7, pp. 3589-3596, Jul. 2013.

[25] D. C.-Serrano, J. S. G.-Diaz, J. P.-Carrier, and A. A. Melcon, "Spatially dispersive graphene single and parallel plate waveguides: analysis and circuit model," IEEE Trans. Microw. Theory Tech., vol. 51. no. 12, pp. 4333-4344, Dec. 2013.

[26] K. Sankaran, "Accurate domian truncation techniques for time-domain conformal methods," Ph.D. dissertation, Dept. Inform. Technol. Elect. Eng., ETH Zurich, Zurich, Switzerland, 2007.

[27] J. M. Jin, The Finite Element Method in Electromagnetics, 2nd ed. New York, NY, USA: Wiley, 2003.

[28] P. Li and L. J. Jiang, "Integration of arbitrary lumped multiport circuit networks into discontinuous Galerkin time-domain analysis," IEEE Trans. Microw. Theory and Techn., vol. 61, no. 7, pp. 2525-2534, Jul. 2013.

[29] S. D. Gedney, C. Luo, J. A. Roden, R. D. Crawford, B. Guernsey, J. A Miller, T. Kramer, and E. W. Lucas, "The discontinuous Galerkin finite element time-domain method solution of Maxwell's equations," J. Appl. Comput. Electromagn. Society, vol. 24, no. 2, pp. 129-142, Apr. 2009.

[30] J. S. Hesthaven and T. Warburton, Nodal Discontinuous Galerkin Methods. Berlin: Springer, 2008.

[31] B. Q. Li, Discontinuous Finite Elements in Fluid Dynamics and Heat Transfer, Springer, 2006.

[32] M. J. Grote, M. Mehlin, and T. Mitkova, "Runge-Kutta based explicit local time-stepping methods for wave propagation," University of Basel, Preprint, 2014.

[33] J. Niegemann, R. Diehl, and K. Busch, "Efficient low-storage RungeKutta schemes with optimized stability regions," J. Comput. Phys., vol. 231, no. 2, pp. 364-372, Jan. 2012. 